\title{
Haematocrit, age and survival in a vertebrate population
}

\author{
Thomas Brown ${ }^{1}$, Martijn Hammers ${ }^{2}$, Martin Taylor ${ }^{1}$, Hannah Dugdale ${ }^{3}$, Jan Komdeur ${ }^{2}$, \\ and David Richardson ${ }^{1}$ \\ ${ }^{1}$ University of East Anglia \\ ${ }^{2}$ University of Groningen \\ ${ }^{3}$ University of Leeds
}

September 10, 2020

\begin{abstract}
Understanding trade-offs in wild populations is difficult, but important if we are to understand the evolution of life histories and the impact of ecological variables upon them. Markers that reflect physiological state and predict future survival would be of considerable benefit to unravelling such trade-offs and could provide insight into individual variation in senescence. However, currently used markers often yield inconsistent results. One underutilised measure is haematocrit, the proportional of blood comprising of erythrocytes, which relates to the blood's oxygen-carrying capacity and viscosity, and to individual endurance. Haematocrit has been shown to decline with age in cross-sectional studies (which may be confounded by selective appearance/disappearance). However, few studies have tested whether haematocrit declines within-individuals or whether low haematocrit impacts survival in wild taxa. Using longitudinal data from the Seychelles warbler (Acrocephalus sechellensis), we demonstrated that haematocrit increases with age in young individuals $(<1.5$ years $)$ but decreases with age in older individuals (1.5-13 years). In breeders, haematocrit was higher in males than females and varied relative to breeding stage. High haematocrit was associated with lower survival in young individuals, but not older individuals. Thus, while we did not find support for haematocrit as a marker of senescence, high haematocrit is indicative of poor condition in younger individuals. Possible explanations are that these individuals were experiencing dehydration and/or high endurance demands prior to capture, which warrants further investigation. Our study demonstrates that haematocrit can be an informative metric for life-history studies investigating trade-offs between survival, longevity and reproduction.
\end{abstract}

\section{Hosted file}

Haematocrit_draft_for_submission_E\&E.pdf available at https://authorea.com/users/357761/ articles/480194-haematocrit-age-and-survival-in-a-vertebrate-population 